International Journal of Quantum Information

(C) World Scientific Publishing Company

\title{
Numerical characterization of atmospheric effects on an Earth-Space quantum communication channel.
}

\author{
N. Antonietti *, M. Mondin \\ Dipartimento di Elettronica, Politecnico di Torino, Corso Duca degli Abruzzi, 24, (TO) - Italy \\ G. Brida, M. Genovese † \\ INRIM; strada delle Cacce 91, 10135 Torino, Italy
}

Received 30-05-2006

\begin{abstract}
Quantum communication in free space is the next challenge of telecommunications. Since we want to determine the outcome of quantum communication by means of single photons, we must understand how a single photon interacts with the atmosphere. In this brief article, some simulation results for realistic and generic atmospheric conditions are reported, a related experiment is considered and its results are described and discussed.

Keywords: entangled states; quantum communication.
\end{abstract}

\section{Introduction}

Quantum Key Distribution (QKD) has now reached substantially a commercial level having been realized up to many tens kilometer both in fiber and open space 1 .

Nowadays the frontier of QKD is the realization of a Earth-Space or a SpaceSpace quantum communication channel $7 / 9[10 / 11$.

This realization would be of utmost relevance both for quantum key distribution 1 , since it would allow intercontinental quantum transmissions, and for studies concerning foundations of quantum mechanics 788 . Thus, preliminary feasibility studies have been performed showing its practical realizability.

In little more details, in ref. 9 a BB84 scheme was studied for earth-Space communication. By considering gaussian optics, a $15 \mathrm{~dB}$ loss was attributed to diffraction, whilst aerosol loss was considered of secondary relevance (0.04-0.06 dB) for transmission with clear sky and from high elevation above sea. Altogether atmospheric losses were estimated to be about 2-5 dB. On the other hand, the security level for quantum transmission was estimated to be $40 \mathrm{~dB}$, lowering to $10 \mathrm{~dB}$ if

*nicolo.antonietti@polito.it

†genovese@inrim.it 
the eavesdropper (conventionally dubbed Eve) had technologies for intercepting selectively an eventual multi-photon component.

In another study, ref. ${ }^{7}$, a $6.5 \mathrm{~dB}$ loss was estimated by considering optics and finite quantum efficiency of detectors. The limit for secure quantum transmission was estimated to be at $60 \mathrm{~dB}$ loss. Here atmospheric losses were estimated to be around $1 \mathrm{~dB}$.

Finally, a more systematic study was performed in 16 by using a program of US Airforce. Nevertheless, no detail was given of the code and few results were really presented (atmospheric losses were estimated to be $1 \mathrm{~dB}$ ).

Effectively, daylight and open space transmission above $10 \mathrm{~km} 15$ and at a distance of up to $23 \mathrm{~km} 14$ were performed, and an European collaboration is preparing an experiment at Canary islands for a distance up to $120 \mathrm{~km}$.13.

All these theoretical and experimental studies guarantee the feasibility of ground-space channel. Nevertheless, the analysis of atmospheric effects is rather incomplete and is far from considering various realistic atmospheric situations that could be met during a real transmission. Even in the very general review of ref. 16 , only few results are presented, and with small detail.

Thus, a detailed analysis of atmospheric effects in various realistic situations would be of the utmost relevance.

Purpose of this paper is to describe a work addressed to reach a precise characterization of atmospheric effects on a quantum communication channel by using a well tested simulation program.

To investigate this topic we have used a free source library for radiative transfer calculations named libRadtran 2 . This library can solve the radiative transfer equations, set some input parameters and exploit the HITRAN 3 database, which is a high-resolution atmospheric parameters database.

In our simulations we can determine what part of the solar radiation is the direct downward irradiance. However, we are still not able to say anything about the possible photon depolarization (whose precise estimation is still in progress).

Various parameters can influence the atmospheric effects on the photon transmission, as, for instance, aerosols, pressure, temperature, air density, precipitations, cloud composition, humidity, chemical components. As a first step in order to evaluate their relevance in various meteorological conditions, here we present some preliminary results obtained by varying one of them at time.

\section{Real experiments}

\subsection{Different distributions of aerosols}

In libRadtran, a database of aerosols distributions can be found. It has been written according to ref. 4 . There, four aerosols distributions for four different environment conditions are described (rural, maritime, urban, tropospheric).

For our first analysis, the atmospheric conditions are selected to be in summer, 
at midlatitudes, according to ref. 5 and the source irradiance is chosen in accordance to ref. 6. both databases are present in libRadtran.

For these conditions the downward direct irradiance at the Earth surface with the source at the zenith outside the whole atmosphere has been evaluated. We chose the visible wavelength because this is the range used in quantum communications and is not affected by strong absorption as UV and IR bands; actually the exact range is from $256 \mathrm{~nm}$ to $1010.320 \mathrm{~nm}$.

A first result of this analysis is that this quantity is largely independent on the aerosol type, as it can be observed in figure (1).

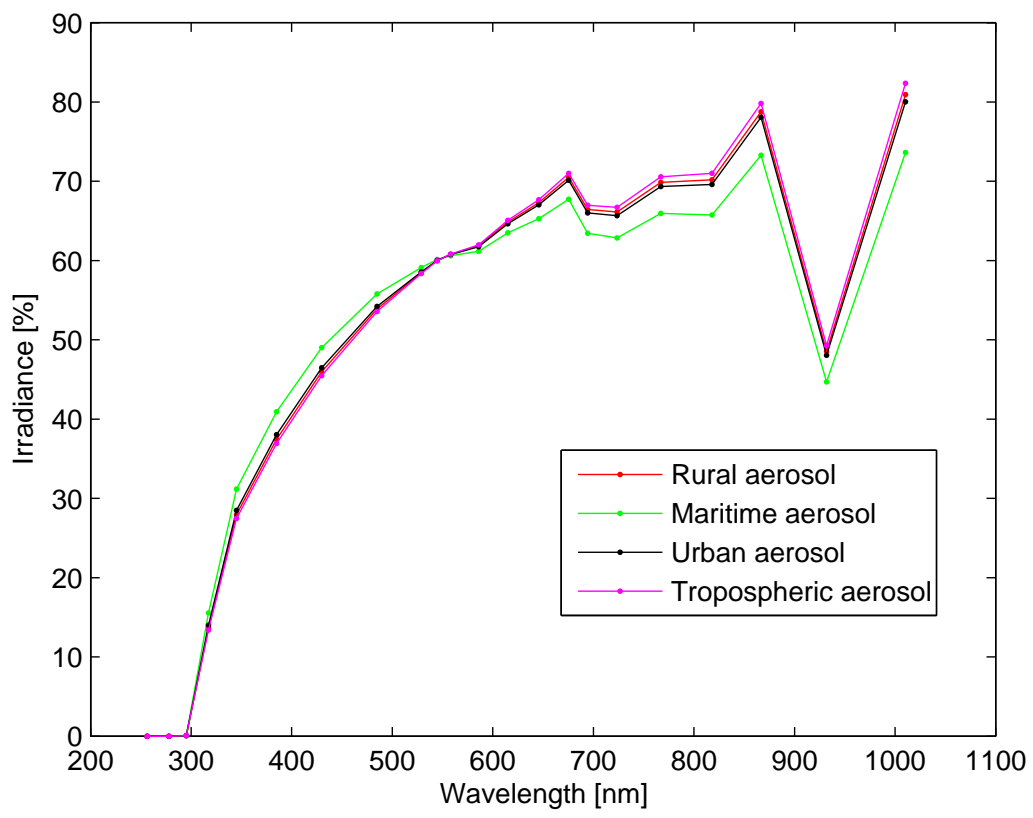

Fig. 1. Ratio of direct downward irradiance with respect to the source irradiance, in the visible range, for different aerosols conditions

Ut can also be observed that the best range for communications is roughly from $700 \mathrm{~nm}$ to $900 \mathrm{~nm}$. In this range, the fraction source light which gets across the atmosphere without any interaction with the atmosphere is about $75 \%$ (i.e. a photon has a $75 \%$ probability to get across the model of atmosphere we have built without interacting with it). 


\subsection{Different temperature profile}

In the considered atmosphere database, the temperature increases fairly linearly from the ground level value $T_{0}$ up to $15 \mathrm{~km}$, where it assumes a given value $T_{15}$. $T_{0}$ is actually a free parameter, and we have varied its value from $-10^{\circ} \mathrm{C}$ up to $30^{\circ} \mathrm{C}$, with steps of $5^{\circ} \mathrm{C}$. The air density is modified automatically, by the program, according to the perfect gas law. Above $15 \mathrm{~km}$, the parameters have been left unchanged, and no aerosols presence has been considered. The obtained results are depicted in figure (2).

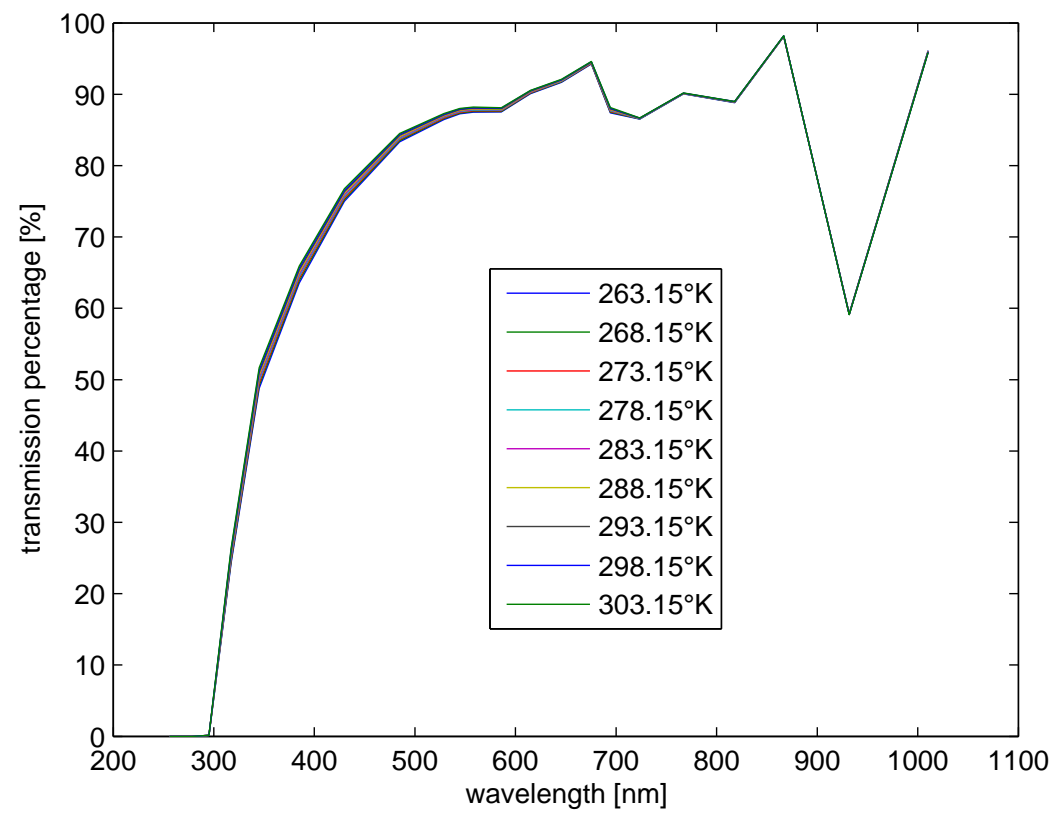

Fig. 2. Ratio of direct downward irradiance with respect to the source irradiance, in the visible range, for different temperature profiles

Once again, it is possible to observe that the transmissivity is not affected by the above modifications; and the best range for the communications is roughly from $700 \mathrm{~nm}$ to $900 \mathrm{~nm}$ as well.

\subsection{Different humidity profiles}

In order to observe the effect of humidity, a further modification has been added to the atmospheric conditions of ref. 5 . This time, the relative humidity has been set as a constant value in the first $15 \mathrm{~km}$ of the atmosphere. The values are $5 \%$ and from $10 \%$ to $100 \%$ with steps of $10 \%$. No aerosols have been considered in this 
configuration. In figure (3), the obtained results can be observed.

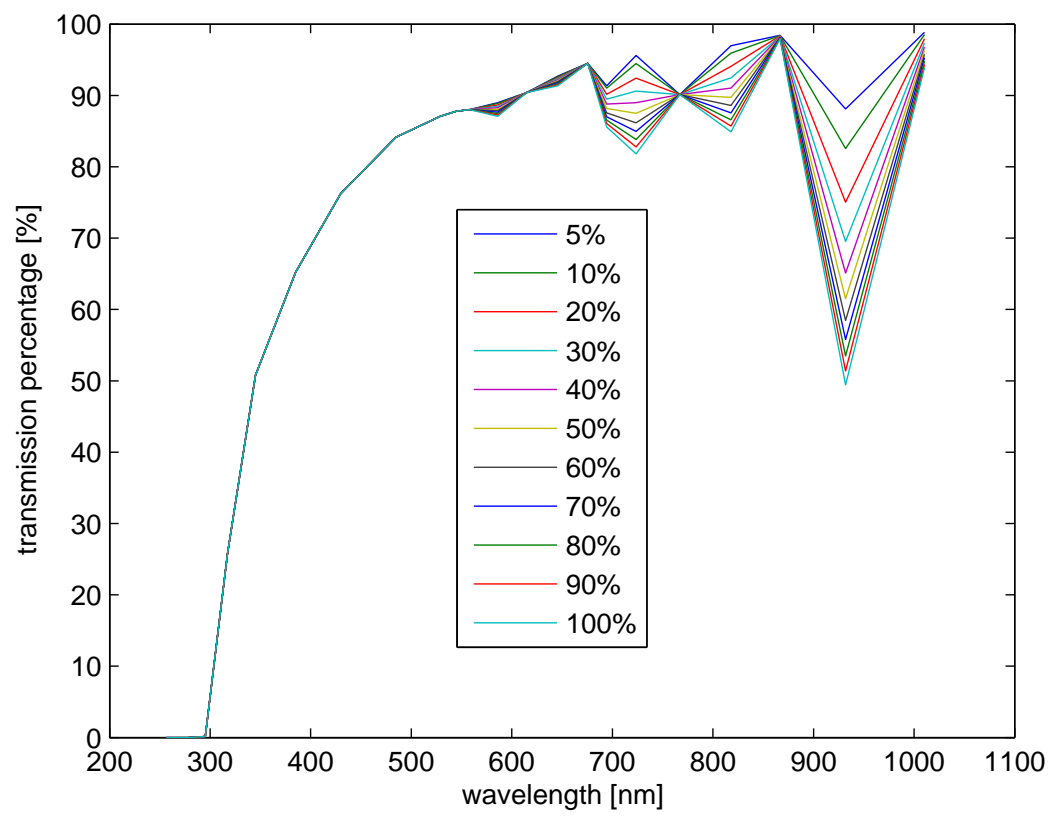

Fig. 3. Ratio of direct downward irradiance with respect to the source irradiance, in the visible range, for different relative humidity profiles

Also in this scenario, the most advantageous range for communication is from $700 \mathrm{~nm}$ to $900 \mathrm{~nm}$, but in this case we can observe some differences among different humidity conditions. As it can be observed from figure (3), the HITRAN $\sqrt{3}$ database, being based on measurements performed with wave-packets and not with photons, has, in some regions, a somewhat low resolution with respect to what would be needed for our application. As a consequence, some low-resolutions effects, like those observed in the $700 \mathrm{~nm}$ to $1000 \mathrm{~nm}$ region, can arise. The absorption in the range between $800 \mathrm{~nm}$ and $1000 \mathrm{~nm}$ is water vapour dependent and is strongly affected by its presence. These preliminary results point out the necessity of a more precise analysis with an increased resolution in wave length.

\subsection{Presence of clouds}

In order to study the possibility of establish a quantum communication channel, the presence of clouds has to be considered as well. In order to do this, we have added clouds to the atmosphere 5 without aerosols. The model we use describes the clouds as two-dimensional objects, without depth. We considered different cloud 
configurations, inserting clouds at 2,3 km, at 2,3,4 km and at 2,3,4,5 km. In our model, every cloud has a liquid water content of $1 \mathrm{gm}^{-3}$ and an effective droplet radius of $10 \mu \mathrm{m}$. The results are presented in figure (4).

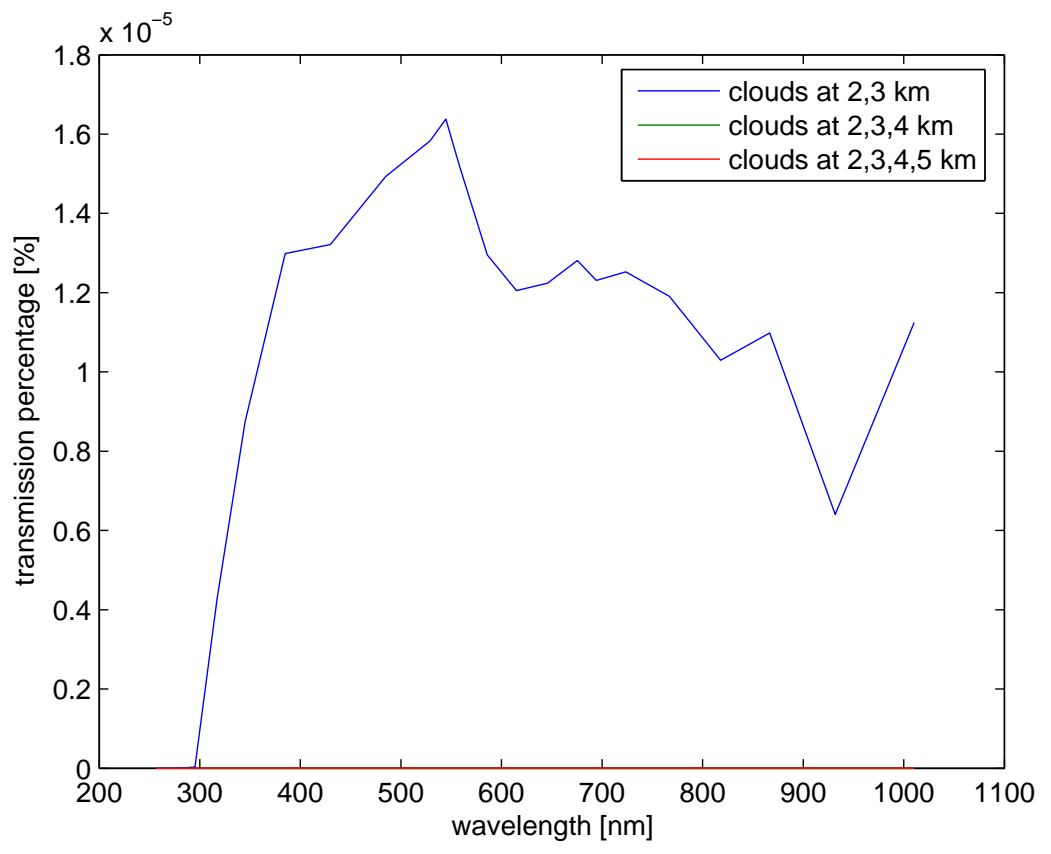

Fig. 4. Ratio of direct downward irradiance with respect to the source irradiance, in the visible range, for different cloud configurations

It can be immediately observed that the presence of clouds seriously harms the quantum communication. Only the configuration with two layers of clouds at 2 $\mathrm{km}$ and $3 \mathrm{~km}$ gives a transmission percentage value different from zero, actually the transmissivity values are in the order -5 , and therefore not suitable for our application. Notice that the selected cloud configurations belong to the set contained within the libRadtran database, but also other configurations could be considered, if needed.

\subsection{Comparison between two extremely different conditions}

Finally we want to get an idea of how is transmissivity for two extremely different conditions. On one side there is a city environment with relevant aerosols concentrations and $90 \%$ relative humidity, on the other side a dry desert without aerosols. The results for these two cases are depicted in figure (5).

As we could expect, a dry desert is a much better environment for quantum 


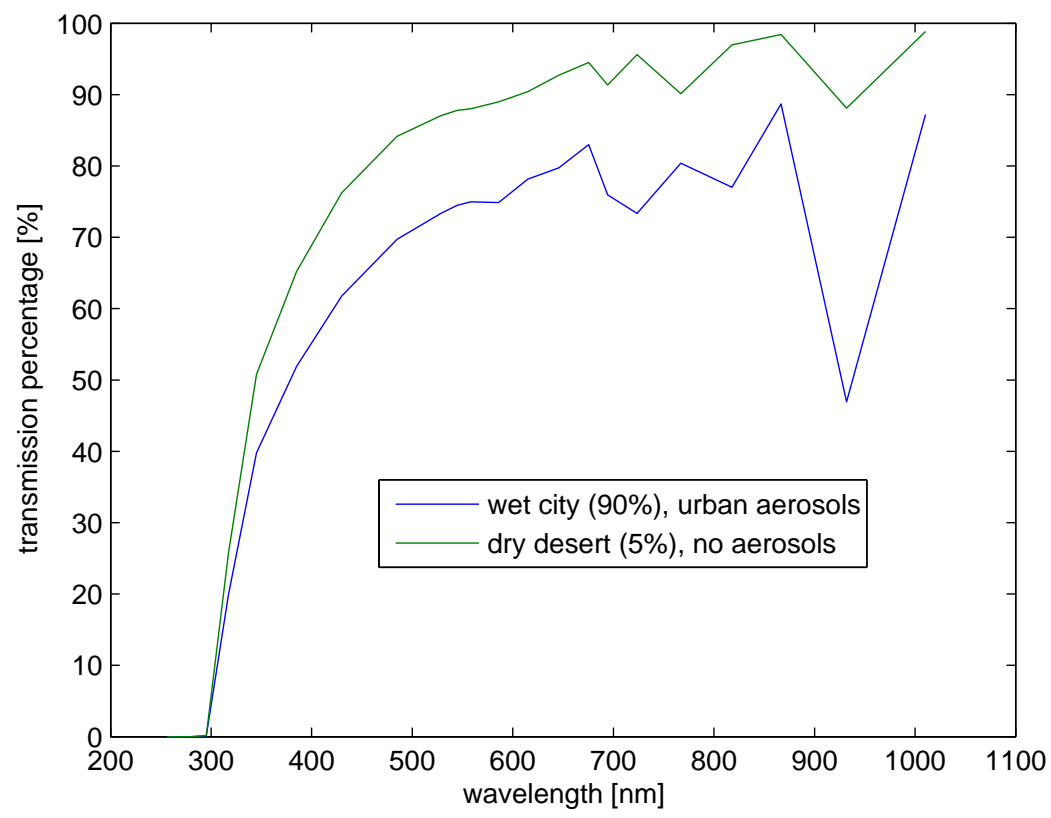

Fig. 5. Ratio of direct downward irradiance with respect to the source irradiance, in the visible range, for two extremely different environments

communication than a humid city. Nevertheless, also in this second case losses are not such to compromise the possibility of realizing a secure QKD.

\section{Conclusions}

If we want to investigate atmospheric effects on a quantum channel, with the purpose of predicting the possible results of an experiment, we have to solve stochastic equations. In this paper we have presented some preliminary results obtained by using the free source library libRadtran. Our results show that a further deeper analysis of atmospheric effects based on this approach could effectively be a useful tool for predicting the performances of a quantum communication channel in various realistic operative meteorological situations.

\section{References}

1. N. Gisin, Rev. Mod. Phys. 74 (02) 145.

2. http://www.libradtran.org/

3. http://cfa-www.harvard.edu/hitran/

4. E. P. Shettle, Models of aerosols, clouds and precipitation for atmospheric propagation studies, "Atmospheric propagation in the UV, visible, ir and mm-region and related system aspects", vol. 454, (1989) 
5. G. P. Anderson, S. A. Clough,F. X. Kneizys, J. H. Chetwynd and E. P. Shettle, AFDL atmospheric constituent profiles, "AFGL Tech. Rep., AFGL-TR-86-0110", Air Force Geophysical Laboratories (1986).

6. S. Kato and T. P. Ackerman and J. H. Mather and E. E. Clothiaux, The k-distribution method and correlated-k approximation for a shortwave radiative transfer model, "J. Quant. Spectrosc. Radiat. Transfer", vol. 62 pp. 109-121, (1999).

7. R. Kaltenbaeck et al., "Quantum Comm. and Quantum Imag.", ed R. Meyers and Y. Shih, Proc. of SPIE 5161, pag. 252. P. Villoresi et al., quant-ph 0408067. M. Aspelmeyer et al., IEEE Sel. Top. Quant. El. 9 (03) 1541.

8. M. Genovese, Physics Reports 413/6 (2005) 319.

9. J.. Rarity et al., "Quantum Comm. and Quantum Imag.", ed R. Meyers and Y. Shih, Proc. of SPIE 5161, pag. 240.

10. P.J. Edwards et al., "Quantum Comm. and Quantum Imag.", ed R. Meyers and Y. Shih, Proc. of SPIE 5161, pag. 152.

11. G. Catastini, M. Rasetti, R. Ionicioiu, G. Brida, M. Genovese, Communication presented at ONERA workshop, Paris, April 2005.

12. G. Gilbert and M. Hamrick, quant-ph0009027

13. T. Occhipinti, personal communication.

14. C. Kurtsiefer et al., Nature 419 (02) 450.

15. C. Peng et al., Phys. Rev. Lett. 94 (05) 150501; R.J. Hughes et al., La-UR-02-449; R. Alleaume et al., quant-ph 0402110

16. G. Gilbert and M. Hamrick, quant-ph 0009027

17. Aspelmeyer A. et al., Long-Distance Free-Space Distribution of Quantum Entanglement, vol. 301, no. 5633, (2003) 